Artigo Original

Original Article

Suelen Bernardo Guckert ${ }^{1}$

Carolina Rogel de Souza ${ }^{1}$

Aline Megumi Arakawa-Belaunde ${ }^{1}$ (B)

Descritores

Promoção da saúde

Fonoaudiologia

Atenção Básica

Saúde da Família

Pessoal de Saúde

Keywords

Health Promotion

Speech-Language Sciences

Primary Health Care

Family Health

Health Personnel

Endereço para correspondência: Aline Megumi Arakawa-Belaunde Centro de Ciências da Saúde,

Coordenadoria Especial de

Fonoaudiologia, Campus Universitário,

Trindade-Florianópolis (SC), Brasil.

E-mail: arakawa.aline@ufsc.br

Recebido em: Abril 02, 2019.

Aceito em: Outubro 14, 2019.

\section{Atuação fonoaudiológica na atenção básica na perspectiva de profissionais dos núcleos de apoio à saúde da família}

\author{
The role of speech-language therapists in primary \\ healthcare from the perspective of professionals \\ in family health support centers
}

\begin{abstract}
RESUMO
Objetivo: Analisar a percepção dos profissionais que compõem os Núcleos de Apoio à Saúde da Família (NASF) de uma capital da região sul do País, sobre a atuação do fonoaudiólogo na atenção básica (AB). Método: Trata-se de um estudo qualitativo, observacional transversal. Participaram da presente pesquisa todos os profissionais do quadro do NASF que aceitaram voluntariamente colaborar com a pesquisa. Aplicou-se um questionário semiestruturado contendo informações sobre o perfil profissional e suas atividades desenvolvidas na AB. Resultados: Os profissionais de saúde realizam ações que possibilitariam a participação conjunta do fonoaudiólogo, porém, essa especialidade não está contemplada no quadro de profissionais que integram as equipes do município estudado. De acordo com o questionário, os encaminhamentos para a fonoaudiologia acontecem principalmente em assuntos relacionados à fala e linguagem infantil. Percebe-se pouco conhecimento acerca da contribuição fonoaudiológica no que diz respeito às ações interdisciplinares. Conclusão: Os participantes deste estudo demonstraram uma percepção reducionista no que diz respeito à atuação fonoaudiológica na $\mathrm{AB}$. Além disso, as ações realizadas por estes profissionais direcionam-se àquelas que o fonoaudiólogo poderia contribuir. Sugere-se que próximos estudos possam demonstrar os benefícios que o fonoaudiólogo pode levar para as atividades realizadas.
\end{abstract}

\begin{abstract}
Purpose: To analyze the perception of professionals constituting Family Health Support Centers (FHSC) of a capital in the South Region of Brazil regarding the role of speech-language therapy professionals in primary care (PC). Method: This is a qualitative, observational cross-sectional study. All professionals constituting the FHSC who voluntarily agreed to collaborate with the research participated in this study. A semi-structured questionnaire was applied containing information about the professional profile and activities developed in PC. Results: Health professionals perform actions that would allow for the joint participation of speechlanguage therapy professionals, since this category is not contemplated in the staff members that constitute the municipality team analyzed. According to the questionnaire, referrals to speech-language therapy professionals are mainly related children issues with speech and language. Little knowledge on the contribution of speechlanguage sciences regarding interdisciplinary actions was found. Conclusion: The participants of this study demonstrated a reductionist perception regarding speech-language sciences in PC. In addition, the actions performed by the professionals of this study are directed towards what the speech-language professional could contribute instead. Further studies could demonstrate the benefits that speech-language professionals can provide to the activities performed.
\end{abstract}

Trabalho realizado no Departamento de Fonoaudiologia da Universidade Federal de Santa Catarina UFSC - Florianópolis (SC), Brasil.

${ }^{1}$ Universidade Federal de Santa Catarina - UFSC - Florianópolis (SC), Brasil.

Conflito de interesses: Nada a declarar.

Fonte de financiamento: Nada a declarar. 


\section{INTRODUÇÃO}

O Sistema Único de Saúde (SUS) foi criado com o objetivo de oferecer à população o reconhecimento da saúde como direito social contemplado por políticas públicas de modo universal e integrado, gerando reflexões sobre o olhar direcionado à atenção à saúde ${ }^{(1)}$. Em 1994, para fortalecer esse novo modelo de atenção à saúde, foi criado o Programa de Saúde da Família (PSF), posteriormente denominado de Estratégia de Saúde da Família (ESF) e, no ano de 2008, o Núcleo de Apoio à Saúde da Família (NASF) ${ }^{(2)}$.

Em 2017, ocorreram algumas modificações no modelo adotado pelo NASF. A principal foi em relação às equipes apoiadas, pois, atualmente, o NASF também auxilia equipes de atenção básica (AB) denominadas tradicionais, mudando sua nomenclatura para Núcleo Ampliado de Saúde da Família e Atenção Básica (NASF-AB). A composição das equipes NASF-AB não sofreu nenhuma alteração, permanecendo com diferentes profissões e especialidades da área da saúde atuando de maneira integrada ${ }^{(3)}$.

Dado o que se precede, pode-se citar a atuação da fonoaudiologia desde a implementação do SUS, ganhando espaço ao se inserir na $\mathrm{AB}^{(4)}$. Junto à equipe multidisciplinar, o profissional pode realizar atividades como o diagnóstico situacional e institucional, o acolhimento, as visitas domiciliares, os atendimentos individualmente e/ou em grupos, ações de educação em saúde no interior (salas de espera) e/ou no entorno das unidades de saúde (grupos de atividades físicas, de cuidados em saúde e de convivência, de saúde mental, de artes, matriciamento, atuação em campanhas de saúde, educação permanente das equipes, bem como a realização e divulgação de pesquisas) ${ }^{(2)}$. Destaca-se que, quando inserido na equipe do NASF, o fonoaudiólogo deve priorizar as atividades de cunho coletivo, colaborando para o fortalecimento do apoio social e aproximação da comunidade ${ }^{(5)}$.

Existem demandas exclusivas do profissional fonoaudiólogo para o atendimento clínico e coletivo, para atuar de maneira reabilitadora e, também, realizando a prevenção e promoção da saúde ${ }^{(6)}$.

O enfoque dado em prol da promoção da saúde pode ser enfatizado no conjunto de ações desenvolvidas pelos profissionais da $\mathrm{AB}$, oferecendo à comunidade uma rede de apoio com estratégias de promoção de saúde adequadas, em que toda a rede seja contemplada, atendendo às necessidades da população(7). De acordo com a Política Nacional de Promoção da Saúde, é fundamental a participação dos demais setores e instituições para a produção de saúde e do cuidado ${ }^{(8)}$.

As ações promotoras de saúde contribuem para uma melhor qualidade de vida da população ${ }^{(8)}$. Além disso, os atendimentos coletivos promovem um ambiente favorável à troca de experiências e vivências aos participantes, bem como possibilita a adoção de hábitos de vida saudáveis, que refletirá no bem-estar desses indivíduos $^{(6)}$.

Nesse caso, destaca-se a importância de seguir os princípios da $\mathrm{AB}$, que tange pela promoção e prevenção à saúde, na integralidade do serviço/atendimento prestado ${ }^{(7)}$. Nascimento e Nakamura (2018)(4) relataram, em seu estudo, que essa carência do profissional fonoaudiólogo na equipe de saúde é reflexo da má distribuição e recursos humanos insuficientes destinados à área da saúde, gerando impacto negativo para a comunidade, como falta de acesso, além do conhecimento, que pode se tornar restrito, sobre as possíveis atuações da Fonoaudiologia ${ }^{(9)}$.

Dessa forma, o presente estudo tem por objetivo analisar a percepção dos profissionais que compõem os Núcleos de Apoio à Saúde da Família, de uma capital da região sul do País, sobre a atuação do fonoaudiólogo na $\mathrm{AB}$.

\section{MÉTODO}

Trata-se de um estudo de cunho qualitativo, observacional transversal desenvolvido com profissionais pertencentes ao NASF de uma capital da região sul do País. Foram incluídos todos os profissionais que compõem o quadro do NASF que aceitaram voluntariamente participar da pesquisa e, excluídos, aqueles afastados do trabalho ou em período de férias no momento da coleta de dados. Salienta-se que o fonoaudiólogo não compõe as equipes NASF do município, no entanto, está presente na médica complexidade e na rede hospitalar.

Inicialmente foi realizado contato com o coordenador de cada distrito sanitário do município para esclarecer sobre os propósitos da pesquisa e viabilizar a participação em reuniões junto às equipes do NASF. Posteriormente, foram agendados dia e horário ideais para que a pesquisadora participasse da reunião a fim de esclarecer os aspectos inerentes à pesquisa em questão aos profissionais participantes.

Para a coleta de dados, aplicou-se um questionário semiestruturado (questões abertas e fechadas), contendo informações sobre o perfil profissional, atividades desenvolvidas como membro do NASF e conhecimento acerca da atuação do fonoaudiólogo.

Os profissionais foram convidados pela pesquisadora, em reunião do NASF, para participar de forma voluntária da presente pesquisa. Aqueles que aceitaram assinaram o termo de consentimento livre e esclarecido após a leitura.

Os dados foram apresentados de forma descritiva e, para a análise e interpretação dos aspectos das questões discursivas, optou-se por utilizar a modalidade temática ${ }^{(10)}$. Após a transcrição, realizou-se a leitura do material pelos pesquisadores envolvidos, iniciando a pré-análise e a exploração dos dados, os quais, posteriormente, foram agrupados em subcategorias temáticas e, por último, categorizados.

Este trabalho faz parte do projeto maior denominado "Promoção de saúde: possibilidades de atuação fonoaudiológica na atenção básica" e encontra-se aprovado pelo Comitê de Ética em Pesquisa com Seres Humanos da instituição de origem, sob o protocolo CAAE n.: 57795116.1.0000.0121.

\section{RESULTADOS}

Este estudo contou com a participação de 39 profissionais que compõem o NASF, referente aos cinco distritos sanitários. Cada NASF possui em média oito profissionais, com cinco e dez componentes no mínimo e máximo, respectivamente. A média etária destes profissionais é de 31 anos $(\mathrm{dp}=15)$, com mínimo e máximo de 23 e 54 anos, respectivamente. A maioria (89,74\%) pertencendo ao gênero feminino, como apresenta a Tabela 1. 
Tabela 1. Perfil dos profissionais que compõem as equipes do NASF

\begin{tabular}{lcc}
\hline Gênero & N & $\%$ \\
\hline Feminino & 35 & $89,75 \%$ \\
Masculino & 4 & $10,25 \%$ \\
\hline Graduação & $\mathbf{N}$ & $\%$ \\
\hline Educação física & 7 & $17,94 \%$ \\
Fisioterapia & 7 & $17,94 \%$ \\
Nutrição & 7 & $17,94 \%$ \\
Psicologia & 6 & $15,38 \%$ \\
Serviço social & 5 & $12,82 \%$ \\
Farmácia & 4 & $10,25 \%$ \\
Medicina & 3 & $7,69 \%$ \\
\hline Escolaridade & $\mathbf{N}$ & $\%$ \\
\hline Lato Sensu & 22 & $51,28 \%$ \\
Stricto Sensu & 12 & $30,76 \%$ \\
\hline Região de formação (graduação) & $\mathbf{N}$ & $\%$ \\
\hline Sul & 20 & $51,30 \%$ \\
Nordeste & 1 & $2,60 \%$ \\
Sudeste & 3 & \\
\hline & & \\
\hline
\end{tabular}

De acordo com a Secretaria Municipal de Saúde do município em referência, no período do desenvolvimento da pesquisa (março a agosto de 2017), o sistema organizacional da Atenção Primária era composto por 50 Centros de Saúde, subdivididos em cinco distritos sanitários, que se responsabilizam pela administração da rede em nível regional: Continente, Centro, Leste, Norte e Sul. Com relação ao NASF, há 12 equipes, assim distribuídas: três no Distrito Continente, três no Distrito Sul, dois no Distrito Centro, dois no Distrito Leste e dois no Distrito Norte (CNES, 2016) ${ }^{(11)}$.

Na composição das equipes dos NASFs, destacam-se os profissionais com formação em Educação Física, Fisioterapia e Nutrição, correspondendo a 17,94\% cada, sendo que 51,30\% referiram a região sul como local de formação. A titulação máxima citada pelos participantes foi lato sensu $(51,28 \%)$, com tempo de atuação profissional no SUS em média de sete anos (mínimo 12 e máximo 21 anos).

Estes profissionais estão atuando no NASF em média há 56 meses (mínimo 12 e máximo 96 meses). Cada equipe NASF apoia em média 12 ESF (mínimo três e máximo $32 \mathrm{ESF}$ ) e, em média, cinco centros de saúde (mínimo um e máximo $15 \mathrm{CS}$ ).

$\mathrm{Na}$ análise das questões abertas, verifica-se que a contribuição do profissional fonoaudiólogo na AB foi citada por $38(97,44 \%)$ profissionais.

A partir da análise das respostas dos profissionais do NASF, pode-se categorizar em (I) Contribuição da fonoaudiologia na $\mathrm{AB}$, (II) Ações realizadas na $\mathrm{AB}$ com o apoio do profissional do NASF, (III) Grupos de promoção e prevenção de saúde realizados na $\mathrm{AB}$.

\section{Contribuição da fonoaudiologia na atenção básica:}

Diante das respostas descritas no questionário, constatou-se que o profissional fonoaudiólogo pode contribuir de diversas maneiras junto aos demais profissionais do NASF. Por exemplo, na realização do matriciamento das equipes, na capacitação dos profissionais da ESF, nas visitas domiciliares, no apoio às equipes, na discussão de caso, na participação das reuniões de equipe, nas ações realizadas pelo PSE e considerando a demanda do Centro de Saúde, como se observa nos trechos abaixo.

"A Fonoaudiologia pode contribuir na AB matriciando as ESF, participando das reuniões do PSE e atendendo às demandas". (P1)

"Apoiando as equipes nas demandas específicas da Fonoaudiologia, assim como ajudando a ampliar o escopo de ações da ESF, contribuindo assim para a promoção da saúde". (P13)

"[...] atividades do NASF como visitas domiciliares, matriciamento junto à ESF [...] Também em todas as atividades que possam ser aprimoradas com os conhecimentos especificos da área". (P19).

"Capacitação da Equipe, matriciamento, diminuir o número de encaminhamentos para a atenção secundária". (P22)

"Matriciamento com as ESFs para discussão de casos, educação permanente junto à ESF". (P37)

A atuação da fonoaudiologia pode ser feita em todos os ciclos de vida, do nascimento até a senescência, por meio de estratégias coletivas e individuais, suprindo as necessidades existentes, sejam elas individuais e/ou coletivas. Além disso, essas ações também são realizadas fora do âmbito do Centro de Saúde, conforme relato abaixo:

"Com ações extramuros em escolas, centro comunitário, empresas”. (P8)

Verificou-se que $16(41,02 \%)$ dos participantes da pesquisa realizaram encaminhamentos para o fonoaudiólogo devido às demandas e necessidades apresentadas pelo paciente, diante de uma ampla gama de patologias que incidiram principalmente no aspecto relacionado à fala e linguagem infantil.

"Avaliação de transtorno de aprendizagem, desenvolvimento da linguagem, investigações de TDAH, gagueira, mutismo, investigar problema de audiçãolfala". (P2)

"Problemas de linguagem, aprendizagem, de leitura/escrita, dificuldades de comunicação em portadores de deficiência e espectro autista". (P10)

"Crianças com dificuldades de aprendizagem / Melhorar deglutição de pessoa idosa / Melhorar saúde vocal de professor". (P19)

O SUS possui muitas demandas para profissionais de áreas específicas, assim como pode ser observado neste estudo, para o fonoaudiólogo. Abaixo, percebe-se na fala dos participantes que a maior demanda se dá para o público infantil com alterações de linguagem, entretanto, como já foi mencionado, os demais 
públicos também se beneficiariam dos conhecimentos de um fonoaudiólogo.

"[...] percebo a necessidade de estreitar o vínculo com o profissional fonoaudiólogo para discussão de casos complexos no sentido de traçar um plano terapêutico em decorrência de muitos casos, principalmente crianças, de dificuldade de aprendizagem, troca na ordem da linguagem, entre outros casos". (P2)

"A necessidade mais frequente que percebo é em relação aos pacientes que tiveram AVC, com alterações de deglutição e fala [...] problemas relacionados ao desenvolvimento das crianças e à paralisia facial no adulto". (P15)

"[...] crianças com dificuldades de aprendizado, grupos de professores - prevenção da saúde vocal e redução de dores corporais, grupos de cuidadores de idosos - cuidados essenciais às pessoas acamadas. Também em todas as atividades que possam ser aprimoradas com os conhecimentos especificos da área". (P18)

"Orientações para pacientes pós-AVC (e acompanhamento), orientações sobre amamentação, para dificuldades de aprendizagem, orientações e acompanhamento de pacientes com alterações na fala e na deglutição e orientações para pacientes com paralisia facial”. (P33)

"Com capacitação aos profissionais, atendimento e orientação de pacientes com problemas de dicção, problemas na audição". (P39)

\section{Ações realizadas na atenção básica com o apoio do profissional do NASF:}

Dentre diversas ações realizadas na $\mathrm{AB}$ que contam com o apoio de profissionais do NASF, têm-se: a reunião de matriciamento, o atendimento individual compartilhado e específico, o atendimento domiciliar compartilhado e específico, a atividade coletiva compartilhada e específica e a elaboração de materiais de apoio, as rotinas, os protocolos e outras ações de educação permanente, assim como relatado pelos profissionais deste estudo, nas falas que se seguem:

"Matriciamento das ESF, atendimento individual e coletivo, participação nas reuniões de saúde mental e nos grupos de escrita da PSE”. (P2)

"Atendimento individual, matriciamento, visita domiciliar, consulta conjunta, grupos de promoção/recuperação de saúde e educação permanente". (P3)

"Atendimentos individuais e interconsultas, visitas domiciliares, participação em grupos de educação em saúde, atividades educativas na escola, ações de matriciamento, educação permanente com profissionais e etc.". (P6)

"Atendimento individual e em grupo, visitas domiciliares, preceptorias de residentes, matriciamento com as ESFs e contatos com a rede intersetorial”. (P18)
"Gerenciamento dos fármacos, consulta farmacêutica, consulta de auriculoterapia, grupo de auriculoterapia, matriciamento com as equipes". (P32)

"Grupos de orientações sobre cuidado, alongamento e prevenção de lesões. Discussão de caso com a ESF, visita domiciliar, atendimentos individuais para avaliação elou acompanhamentos". (P33)

"Matriciamento com as ESFs e discussão de caso, interconsulta com a ESF, participação em grupo com a ESF, atendimento individual e em grupo dos pacientes encaminhados para nutricionista". (P37)

Nessa pesquisa, as ações mais citadas foram respectivamente matriciamento, visita domiciliar e ações em grupos, conforme se constata nas falas dos profissionais participantes. Destaca-se ainda que os membros da equipe NASF, além dos atendimentos prestados na unidade de saúde, podem contribuir também com ações realizadas junto ao PSE e no acolhimento familiar:

"Matriciamento das ESF, atendimento individual e coletivo, participação nas reuniões de saúde mental e nos grupos de escrita da PSE”. (P1)

"Acolhimento, discussão de caso (matriciamento), visita domiciliar, grupo terapêtico”. (P8)

\section{Grupos de promoção e prevenção de saúde realizados na atenção básica}

Entre todos os grupos, os mais mencionados pelos profissionais foram: atividade física, 28,23\%; antitabagismo e grupo de hábitos saudáveis, 9,68\% dos profissionais, respectivamente; e grupo de diabéticos, $8,87 \%$. Os grupos menos citados foram de fisioterapia e vacinação.

Observou-se a prevalência da participação dos profissionais das especialidades de educação física e nutrição. Dentre os pesquisados, sete relataram não participar/desenvolver nenhuma atividade em grupo. Além disso, 82,05\% trouxeram informações referentes à percepção da realização de ações de promoção de saúde executadas em grupos. Do total de 49 menções, sobre a realização de grupos, foram compilados 15 de acordo com a temática apresentada pelos profissionais, conforme mostra a Tabela 2 .

Tabela 2. Descrição das atividades grupais realizadas nos Centros de Saúde, Florianópolis, 2017

\begin{tabular}{lcc}
\hline & $\mathbf{N}$ & $\%$ \\
\hline Profissionais que participam de grupos & 32 & $82,05 \%$ \\
\hline Grupos & $\mathbf{N}$ & $\%$ \\
\hline Atividades Físicas & 35 & $28,23 \%$ \\
Antitabagismo & 12 & $9,68 \%$ \\
Hábitos Saudáveis & 12 & $9,68 \%$ \\
Diabéticos & 11 & $8,87 \%$ \\
Crianças e Adolescentes & 10 & $8,06 \%$ \\
Gestantes & 10 & $8,06 \%$
\end{tabular}


Tabela 2. Continuação..

\begin{tabular}{llc}
\hline & N & $\%$ \\
\hline Redução e Reabilitação de Dores & 9 & $7,25 \%$ \\
Saúde Mental & 6 & $4,83 \%$ \\
Promoção de Saúde em Geral & 5 & $4,03 \%$ \\
Programa Saúde na Escola & 4 & $3,22 \%$ \\
Cuidadores & 3 & $2,41 \%$ \\
Horta Comunitária & 3 & $2,41 \%$ \\
Medicação/Desmedicalização & 2 & $1,61 \%$ \\
Fisioterapia & 1 & $0,80 \%$ \\
Vacinação & 1 & $0,80 \%$ \\
\hline
\end{tabular}

No que diz respeito à ação intersetorial, percebe-se não ser uma prática comum entre os profissionais deste estudo, pois apenas dois a mencionaram como se lê a seguir:

"Grupos de pais e cuidadores, grupo de apoio psicológico, grupo de escuta PSE, matriciamento em saúde mental (discussão de caso, interconsulta), reunião intersetorial, reuniões de NASF e categorias, atendimentos individuais articulados com a RAPS". (P34)

"[...] reunião intersetorial, reuniões de NASF e categorias, atendimentos individuais articulados com a RAPS". (P34)

"Atendimento individual e em grupo, visitas domiciliares, preceptorias de residentes, matriciamento com as ESFs e contatos com a rede intersetorial”. (P18)

\section{DISCUSSÃO}

A presente pesquisa buscou verificar a percepção dos profissionais que compõem os NASFs sobre a atuação do fonoaudiólogo na $\mathrm{AB}$, encontrando prevalência de profissionais do sexo feminino (89,75\%), com a média etária de 31,41 anos $(\mathrm{dp}=15,05)$, aspecto semelhante observado em outros estudos ${ }^{(12,13)}$.

A composição do grupo apresenta maior número de mulheres. Esse aspecto pode estar relacionado às profissões lidarem com o ato de cuidar que, de acordo com a literatura, no passado, as atividades domésticas e o cuidado com família (marido e filhos) eram tarefas exclusivamente atribuídas ao sexo feminino. Observa-se um processo histórico e cultural que com o passar dos anos está se modificando ${ }^{(14)}$.

Importante destacar que a presença do fonoaudiólogo na saúde pública data das décadas de 70,80 , ainda de forma não muito expressiva e desigual no acesso. Com a criação do SUS, ainda na década de 80 , a saúde passa a ser considerada um direito de todo cidadão e a organização do sistema prevê o acesso integral às ações e serviços de saúde. Com o passar dos anos e a crescente discussão sobre a atuação dos profissionais na atenção básica, propostas foram surgindo e hoje a $\mathrm{AB}$ opera sob o modelo da ESF, com foco no território e nas necessidades e demandas desse e de seus habitantes ${ }^{(15)}$.

Assim, em 2008, foi incorporada às ESFs uma nova equipe, com base no apoio matricial, com vistas a aumentar o escopo das ações na AB. Dessa forma, constata-se a criação do NASF, organizado sob a lógica do apoio matricial como já foi falado; da criação e manutenção de projetos terapêuticos singulares em conjunto com as ESFs e os usuários e aposta no redirecionamento do olhar especializado para a clínica ampliada ${ }^{(16)}$.

A composição do NASF é definida de acordo com as necessidades do território e das equipes de saúde e cabe aos gestores municipais estabelecer e distribuir a quantidade de profissionais que irão compor as equipes de cada região ${ }^{(16)}$. A presença do fonoaudiólogo como um dos profissionais do NASF ocasionou aumento no número total na $\mathrm{AB}$, mas ainda longe do ideal para a população brasileira e de forma desigual entre as várias regiões do País ${ }^{(17)}$. A distribuição desigual impacta o acesso universal a esse profissional e suas ações de promoção e prevenção de saúde.

Como observado no presente estudo, foram mencionadas formas de se organizar o trabalho das equipes NASF, a sua maioria contemplando ações de matriciamento, visitas domiciliares, ações junto ao PSE, atendimentos em grupos e discussão de casos com as demais equipes. Importante destacar que a formação do fonoaudiólogo ainda se dá com foco na atividade clínica individual, o que pode, por vezes, dificultar a atuação desse profissional na AB. A ESF e o NASF propõem uma organização do trabalho com foco na equipe e na atuação conjunta entre os profissionais, favorecendo a utilização de tecnologias leves e leve-duras na prática clínica ${ }^{(15)}$. Faz-se de extrema importância apostar na formação em serviço, como os cursos de Residência Multiprofissional em Saúde da Família, com a experiência do dia a dia do serviço, e dos encontros com profissionais diversos e usuários ${ }^{(2,6)}$.

Como relatado acima, uma das formas de ação da fonoaudiologia no NASF apontada pelos profissionais entrevistados é a participação no matriciamento. $\mathrm{O}$ apoio matricial ou matriciamento é uma forma de trabalho conjunto entre equipes distintas, em que uma delas é aquela de contato direto com a população usuária do serviço, que a acompanha no cotidiano das práticas de saúde, e a outra está presente para ajudar a pensar e organizar os projetos de cuidado mais benéficos para cada pessoa ou grupo ${ }^{(12)}$.

Dessa forma, o matriciamento é uma importante ferramenta na construção de projetos cuidadores e uma forma de aproximar as equipes no cotidiano do serviço ${ }^{(8.19)}$. Para a fonoaudiologia, é um mecanismo potente para a inserção do profissional dessa área nas práticas coletivas, podendo auxiliar em uma maior discussão sobre sua própria prática clínica, uma vez que como apoiador ele deverá lançar mão de outras formas de trabalho: discussões em equipe, organização de fluxos de atendimento na rede de serviços, entre tantos outros para além da atuação clínica individual ${ }^{(12)}$.

Para isso, uma das ferramentas utilizada pela ESF e demais equipes de $\mathrm{AB}$ é a visita domiciliar. Como visto anteriormente, as visitas domiciliares foram mencionadas como a segunda atividade mais comum entre os profissionais da equipe, aspecto considerado positivo dada a visibilidade desta modalidade de atendimento entre os profissionais que atuam no município estudado. A visita domiciliar é realizada para aquelas pessoas/ famílias que apresentam dificuldades em se locomover até uma unidade de saúde para receber o atendimento necessário, porém, não são todos os profissionais que realizam essa atividade em sua rotina de atendimentos ${ }^{(2)}$. Além disso é uma forma de estar 
no território, conhecer e reconhecer a realidade vivida pelos usuários em seu meio social. Essas informações auxiliam as equipes na proposição dos projetos de cuidado e na ampliação do olhar, beneficiando a clínica ampliada.

O fonoaudiólogo é favorecido pela visita domiciliar, pois é o momento de conhecer as reais condições de moradia e, para além disso, estabelecer as relações e vínculos familiares ou de amizade. Essas informações auxiliam na tomada de decisão sobre qual terapêutica seguir e como adaptar, caso necessário. Mas novamente se esbarra na deficiência na formação desses profissionais, que podem não ter tido contato com tal experiência, (durante sua formação) dificultando a ampliação do olhar para além do biomédico.

Observa-se nas falas destacadas que, ao menos, quatro delas referem à atuação do fonoaudiólogo no cuidado de alterações relativas ao período de alfabetização, citam que o fonoaudiólogo pode atuar junto aos "transtornos de aprendizagem", "TDAH", "alterações de leitura e escrita", além da presença nas ações do PSE. Salienta-se que o diagnóstico e intervenção das alterações de linguagem precisam ser realizadas precocemente uma vez que os primeiros anos de desenvolvimento da criança são essenciais para a aquisição de conteúdos linguísticos ${ }^{(20)}$. Neste sentido, os sinais de alerta de tais alterações devem ser observados com atenção por todos os profissionais que estão envolvidos com o cuidado da população infantil ${ }^{(21)}$.

No que diz respeito à atuação fonoaudiológica em aspectos relacionados à linguagem, verifica-se, em um estudo realizado com os profissionais da $\mathrm{AB}$, que apenas $53,33 \%$ dos participantes conseguiram identificar possíveis alterações de linguagem. Esse mesmo estudo detectou que os profissionais da AB apresentam interesse em aprofundar os conhecimentos sobre o desenvolvimento da linguagem na infância ${ }^{(20)}$. Esse dado é extremamente importante para a fonoaudiologia, uma vez que pode justificar a presença do fonoaudiólogo na $\mathrm{AB}$, seja apoiando as ESFs nas discussões de casos envolvendo alterações de linguagem, seja na proposição de ações de educação permanente junto aos mesmos profissionais.

Com base nestes achados, observa-se a necessidade de ter um fonoaudiólogo realizando um trabalho conjunto com os demais profissionais que compõem essas equipes a fim de partilhar conhecimentos, de forma que possa contribuir para futuras ações a serem realizadas pelas ESFs, visando à prevenção e promoção da comunicação humana ${ }^{(21)}$.

Destaca-se, também, o pequeno número de profissionais, os quais citaram a saúde mental, sendo apenas dois deste estudo. A saúde mental deve ser um dos assuntos abordados nas discussões dos profissionais das equipes, visto que é uma temática fundamental para auxiliar o sujeito a manter um equilíbrio social, bem como possibilita a inserção deste indivíduo na comunidade em que vive. Esta é uma questão que merece discussão a fim de modificar os paradigmas no campo da saúde ${ }^{(22)}$, e melhorar $\mathrm{o}$ atendimento prestado para pessoas com tais necessidades.

$\mathrm{Na}$ equipe NASF, o fonoaudiólogo tem a possibilidade de atuar no contexto familiar e social, apoiando e participando da transformação de assistência à saúde, agindo junto aos demais profissionais da equipe na elaboração de projetos terapêuticos e contribuindo com o fazer da clínica ampliada que considera os indivíduos e suas necessidades ${ }^{(5,9)}$. Diante de tais possibilidades, seria enriquecedor se, no município de estudo, o(s) gestor(es) (re) conhecessem a possibilidade da inserção do fonoaudiólogo junto às equipes em prol da atenção à saúde com caráter resolutivo $\mathrm{e}$ integrado como preveem as diretrizes e fundamentos da $\mathrm{PNAB}^{(3)}$.

Neste contexto, a equipe NASF pode se articular com seus saberes para que juntos possam compartilhar as práticas em saúde ao atender às demandas do território ${ }^{(21)}$. Com a troca de informações e experiências dos participantes do presente estudo, é possível elaborar estratégias que ofereçam à população um atendimento interdisciplinar e de qualidade, envolvendo os profissionais da $\mathrm{AB}$.

A presença do fonoaudiólogo na $\mathrm{AB}$, como já descrito, pode auxiliar na detecção precoce de alterações de linguagem, de audição, na proposição de ações coletivas e intersetoriais focadas no processo de alfabetização; todas essas atividades acontecendo na unidade de saúde, na escola, no território em que estão inseridos. Com a aposta em ações coletivas e compartilhadas na $\mathrm{AB}$, o fonoaudiólogo aumenta o acesso da população às suas ações e diminui o fluxo de atendimentos na atenção secundária, auxiliando na organização da rede de saúde, na otimização de recursos humanos e materiais.

Para que esse compartilhamento de saberes e aprendizados seja realizado, deve haver a comunicação dos profissionais entre si. Sendo assim, o acompanhamento interdisciplinar pode ser visto como forma de promover o diálogo entre os diversos conhecimentos, realizando um intercâmbio mútuo e contínuo, a fim de enfrentar os desafios encontrados ${ }^{(9)}$. Neste estudo, os profissionais relataram fazer essa troca de informações e conhecimento por meio das reuniões e discussões de casos realizadas pelos profissionais da saúde da família.

Além disso, observou-se que apenas dois profissionais mencionaram a participação em ações intersetoriais. As ações intersetoriais devem ser planejadas com o objetivo de integrar as equipes de referência de cada território ${ }^{(3)}$. No entanto, estas se apresentam escassas na rotina dos profissionais de saúde, seguindo um modelo de gerencialismo com ações pouco integrativas e verticalizadas, concentradas no assistencialismo, enfraquecendo um dos fundamentos da Atenção Primária à Saúde (APS), ou seja, o engajamento entre as equipes e população adscrita. Salienta-se que a interdisciplinaridade pode incidir na implementação das ações de promoção da saúde, enfrentamento e modificações dos Determinantes Sociais da Saúde ${ }^{(23)}$.

Para que a rede tenha capacidade de atender às grandes demandas que surgem, as ações de promoção e prevenção em saúde podem ser realizadas por meio do desenvolvimento de grupos. O atendimento direcionado ao coletivo traz muitos benefícios para os participantes, pois neste espaço ocorrem as trocas de informações, experiências, conhecimentos, dentre outros $^{(24)}$. Por meio dos grupos, em algumas situações, é possível reduzir a lista de espera e oferecer acolhimento aos usuários que aguardam o atendimento com o profissional de determinada área/especialidade.

A presença de atividades em grupo nas unidades de saúde é estimulada, como forma de ampliar o acesso, na discussão de temas específicos e, muitas vezes, focados em condições de saúde (hipertensão, diabetes, gestação) ou por grupos 
específicos (idosos, crianças, mulheres). De fato, o grupo é uma potente ferramenta na produção do cuidado, é espaço para compartilhamento de saberes, técnicos e populares e, também, local de acolhimento e formação de redes comunitárias ${ }^{(25,26)}$.

Ainda sobre as atividades integradas, as práticas fonoaudiológicas podem se fazer presentes na realização de grupos em um atendimento enriquecedor, com a possibilidade da troca de conhecimentos e vivências ${ }^{(24)}$. Embora se encontre ênfase nas atuações individuais, como mostra o estudo feito por Andrade et al. $(2014)^{(6)}$, ao verificar que grande parte dos fonoaudiólogos que participaram da sua pesquisa realizaram atendimentos individuais e coletivos, prevaleceram as intervenções realizadas individualmente, considerando as demandas específicas do seu conhecimento.

Alguns grupos com temáticas específicas podem contar com a presença do fonoaudiólogo e sua técnica de forma mais direta, como o grupo antitabagismo, no qual a participação do fonoaudiólogo enriquece os momentos dialógicos dado o impacto do fumar na alteração da voz, nas doenças respiratórias, nos problemas cardíacos e no câncer de cabeça e/ou pescoço ${ }^{(27)}$.

Os grupos de hábitos saudáveis envolvem aspectos de cuidado que poderão contribuir para a prevenção de comorbidades como diabetes mellitus, hipertensão arterial, bem como as doenças cardiovasculares $^{(28)}$. Na literatura, observa-se a dificuldade em encontrar a participação da fonoaudiologia em atividades em grupo, principalmente quanto aos hábitos saudáveis.

A exemplo, tem-se a realização de grupo de diabéticos, o qual auxiliará no controle da doença e orientação à população, pois neste espaço o indivíduo poderá trocar informações e experiências de maneira fluida entre seus pares ${ }^{(29)}$. A fonoaudiologia pode atuar nesta prática, auxiliando no planejamento das estratégias de saúde pública que levarão à modificação do quadro atual de cada paciente e, consequentemente, melhorando a qualidade de vida dessas pessoas, além da redução do custo social ${ }^{(30)}$.

Outros grupos de promoção de saúde mediados pelo fonoaudiólogo podem ser realizados como o grupo de gestantes, abordando assuntos sobre o desenvolvimento da criança, bem como o aleitamento materno; e o grupo de idosos, com o objetivo de oferecer a eles um envelhecimento saudável, a partir da manutenção de suas capacidades funcionais ${ }^{(30)}$.

$\mathrm{Na}$ literatura fonoaudiológica, pouco se discute sobre o processo de desenvolvimento das atividades em grupo com as temáticas relacionadas à fonoaudiologia com a participação de outros profissionais da área da saúde. Verifica-se que as atividades grupais com foco na qualidade de vida são práticas pouco comuns entre as equipes de saúde no que diz respeito à participação e às contribuições do fonoaudiólogo. Vale refletir sobre a necessidade e possibilidade da realização de grupos, pois, por meio desta prática, contribui-se também no aspecto interprofissional, bem como na comunicação interna entre as equipes, em prol do melhor atendimento do paciente.

Como limitações do estudo, salienta-se que durante a coleta de dados encontraram-se algumas dificuldades quanto ao agendamento de data e horário para participar na reunião de cada distrito sanitário, pois elas ocorriam a cada dois meses. Além disso, observou-se que alguns participantes levaram um tempo maior do que o esperado para preencher o questionário, sendo um empecilho em alguns momentos, devido à extensão da pauta das reuniões.

O questionário era composto por questões abertas e fechadas e algumas questões abertas foram entregues com ausência de respostas, outras com informações sucintas e, em alguns momentos, de difícil compreensão. Por fim, alguns profissionais relataram preferir questionários apenas com questões fechadas.

\section{CONCLUSÃO}

De acordo com as análises realizadas a partir das respostas obtidas, os participantes deste estudo demonstraram uma percepção limitada no que diz respeito à atuação fonoaudiológica, assim como a suas contribuições interdisciplinares e intersetoriais envolvendo a AB.

As ações contempladas pelos profissionais participantes da presente pesquisa direcionam-se àquelas que o profissional de fonoaudiologia poderia contribuir, enriquecendo os momentos dialógicos, bem como a resolubilidade das demandas. Neste contexto, faz-se mister o conhecimento por parte da gestão, dado que a contribuição da fonoaudiologia pode ir além do processo (re)habilitador, mas promotor de saúde e de fortalecimento da $\mathrm{AB}$.

$\mathrm{Na}$ literatura, encontram-se poucos estudos acerca da atuação fonoaudiológica em ações concomitantes com outros profissionais da área da saúde, como em momentos vinculados à realização de grupos e ações coletivas.

Sugere-se que próximos estudos possam demonstrar, para as diferentes categorias profissionais e gestoras, os benefícios da presença do fonoaudiólogo na $\mathrm{AB}$, nas ações assistenciais diretas à população, compondo equipes de apoio em conjunto às equipes de $\mathrm{AB}$ e com a ampliação do acesso desse profissional. Tal aspecto pode auxiliar na ressignificação das atividades deste profissional da comunicação, modificando a visão estereotipada restrita à reabilitação que pode ser compreendida equivocadamente, como uma profissão voltada à atenção especializada.

\section{REFERÊNCIAS}

1. Rodrigues PHA. Desafios políticos para a consolidação do Sistema Único de Saúde: uma abordagem histórica. Hist Cienc Saude Manguinhos. 2014; 21(1): 37-60. https://doi.org/10.1590/S0104-59702014000100003

2. Zanin LE, Albuquerque IMN, Melo DH. Speech, language and hearing sciences and the family health strategy: implication of structural dimension in the quality of speech, language and hearing care. ACR. 2015; 20(3): 255-61. https://doi.org/10.1590/2317-6431-2015-1546

3. Brasil, Ministério da Saúde. Portaria n. 2.436, de 21 de setembro de 2017. Aprova a Política Nacional de Atenção Básica, estabelecendo a revisão de diretrizes para a organização da Atenção Básica, no âmbito do Sistema Único de Saúde (SUS). 2017

4. Nascimento CL, Nakamura HY. Fonoaudiologia no Sistema Único de Saúde do Estado de São Paulo. Rev Distúrb Comum. 2018; 30(1): 179-85. https://doi.org/10.23925/2176-2724.2018v30i1p179-185

5. Soleman C, Martins CL. The work of speech therapists under support centers for family health (FHSC) - specificities of primary care. Rev CEFAC. 2015; 17(4): 1241-52

6. Andrade AF, Lima MM, Monteiro NP, Silva VL. Avaliação das ações da Fonoaudiologia no NASF da cidade do Recife. ACR. 2014; 19(1): 52-60. https://doi.org/10.1590/S2317-64312014000100010

7. Malta DC, Reis AAC, Jaime PC, Neto OLM, Silva MMA, Akerman M. O SUS e a Política Nacional de Promoção da Saúde: perspectiva resultados, 
avanços e desafios em tempos de crise. Rev Ciênc Saúde Colet. 2018; 23(6): 1799-809. https://doi.org/10.1590/1413-81232018236.04782018

8. Brasil, Ministério da Saúde. Secretaria de Vigilância à Saúde. Secretaria de Atenção à Saúde. Política Nacional de Promoção da Saúde: PNaPS: revisão da Portaria MS/GM n. 687, de 30 de março de 2006 / Ministério da Saúde, Secretaria de Vigilância à Saúde, Secretaria de Atenção à Saúde. - Brasília: Ministério da Saúde, 2014a

9. Cabrera MFB, Eliassen ES, Arakawa-Belaunde AM. Fonoaudiologia e promoção da saúde: revisão integrativa. Revista Baiana de Saúde Pública. 2018; 42(1): p. 21. https://doi.org/10.22278/2318-2660.2018.v42.n1.a2616

10. Minayo MCS. O desafio do conhecimento: pesquisa qualitativa em saúde. $11^{a}$ ed. São Paulo: Hucitec-Abrasco, 2008

11. CNES. Cadastro Nacional de Estabelecimentos de Saúde. 2016. [acesso em 2016 out 13]. Disponível em: <http://cnes.datasus.gov.br/>

12. Barros JO, Gonçalves RMA, Kaltner RP, Lancman S. Estratégia do apoio matricial: a experiência de duas equipes do Núcleo de Apoio à Saúde da Família (NASF) da cidade de São Paulo, Brasil. Rev Ciênc Saúde Colet. 2015; 20(9): 28-47. https://doi.org/10.1590/1413-81232015209.12232014

13. Reis ML, Medeiros M, Pacheco LR, Caixeta CC. Evaluation of the multiprofessional work of the Family Health Support Center (FHSC). Texto \& Contexto de Enfermagem. 2016; 25(1). https://doi.org/10.1590/0104070720160002810014

14. Giulio RMD, Chun RYS. Impacto da afasia na perspectiva do cuidador. Rev Distúrb Comum. 2014; 26(3): 541-49

15. Sarti TD, Feuerwerker, LCM. Saúde da família em análise: estudo de caso sobre a produção da atenção à saúde de pessoas com diabetes mellitus tipo 2. Revista saúde em redes. 2018; 4: 55-73. https://doi.org/10.18310/244648132018v4n1.1008g238

16. Brasil. Ministério da saúde. Secretaria de Atenção à Saúde. Departamento de Atenção Básica. Núcleo de Apoio à Saúde da Família - Volume I: Ferramentas para a gestão e para o trabalho cotidiano. Cadernos de Atenção Básica, n. 39. Brasília: Ministério da saúde, 2014b. p. 116

17. Viégas LHT, Meira TC, Santos BS, Mise YF, Arce VAR, Ferrite S. Fonoaudiologia na Atenção Básica no Brasil: análise da oferta e estimativa do déficit, 2005-2015, Revista CEFAC. 2018; 20(3):353-62. https://doi. org/10.1590/1982-021620182031918

18. Campos GWS,Domitti AC. Apoio matricial e equipe de referência: uma metodologia para gestão do trabalho interdisciplinar em saúde. Cad Saúde Pública 2007; 23(2): 399-407. https://doi.org/10.1590/S0102311X2007000200016

19. Cunha GT, Campos GWS. Apoio matricial e atenção primária em saúde. Saúde Soc 2011: 20(4): 961-970. https://doi.org/10.1590/S010412902011000400013

20. Pizolato RA, Fonseca LMM, Bastos RS, Fernandes AY, Lefévre F, Maximino LP. Vigilância do desenvolvimento da linguagem da criança: conhecimentos e práticas de profissionais da atenção básica à saúde. Rev CEFAC. 2016; 18(5): 1109-20. https://doi.org/10.1590/1982-0216201618520615

21. Wagner J, Bonamigo AW, Oliveira F, Machado MS. Monitoramento da audição e da linguagem na atenção primária à saúde: projeto piloto. Rev Ciênc Saúde Colet. 2017; 22(11): 3599-06. https://doi.org/10.1590/1413812320172211.30182016

22. Ferreira TPS, Sampaio J, Souza ACN, Oliveira DL, Gomes LB. Produção do cuidado em Saúde Mental: desafios para além dos muros institucionais. Interface - Comunicação, Saúde, Educação. 2017; 21(61):373-84. https:// doi.org/10.1590/1807-57622016.0139

23. Silva DAJ, Tavares MFL. Ação intersetorial: potencialidades e dificuldades do trabalho em equipes da Estratégia Saúde da Família na cidade do Rio de Janeiro. Saúde Debate. 2016; 40(111): 193-05. https://doi.org/10.1590/01031104201611115

24. Freitas CS, Kocourek S, Vey APZ, Foletto HM. Motivação de usuários de uma estratégia de saúde da família em grupos de saúde. Revista Brasileira em promoção da Saúde. 2015; 28(4): 496-503. https://doi. org/10.5020/18061230.2015.p496

25. Nogueira ALG, Munari DB, Fortuna CM, Santos LF. Pistas para potencializar grupos na Atenção primária à Saúde. Rev Bras Enferm [internet]. 2016; 69(5):907-14. https://doi.org/10.1590/0034-7167-2015-0102

26. Menezes KKP, Avelino PR. Grupos operativos na Atenção primária à Saúde como prática de discussão e educação: uma revisão. Cad. Saúde Colet, 2016;24(1):124-30. https://doi.org/10.1590/1414-462X201600010162

27. Santana ADM, Vasconcellos LS, Ribeiro MP. Grupo de tabagismo: uma abordagem interdisciplinar. Cadernos de Educação, Saúde e Fisioterapia. 2017; 4(8)

28. Ferrari TK, Cesar CLG, Alves MCGP, Barros MBA, Goldbaum M, Fisberg RM. Estilo de vida saudável em São Paulo, Brasil. Cad de Saúde Pública. 2017; 33(1): p. 12. https://doi.org/10.1590/0102-311x00188015

29. Mota JMS, Silva AM, Abrel TB, Castro MA, Silva MV, Carvalho-Freitas MN, et al. Redução de ansiedade com grupo de diabéticos: interfaces físicas e psicológicas de uma intervenção. Revista Interinstitucional de Psicologia. 2016; 9(2): 312-23

30. Medeiros EA, Maia RM, Cedro MO, Barbosa MLC, Correia RBF, Tavares PMB, et al. A inserção da fonoaudiologia na estratégia saúde da família: vivências em sobral - CE. Revista de Políticas Públicas. 2009; 8(2):7-15

\section{Contribuição dos autores}

$S B G, A M A B$, contribuíram na concepção, delineamento análise dos dados do estudo, elaboraram as versões preliminares do artigo. CRS e AMAB realizaram a revisão do manuscrito e aprovação final da versão a ser publicada. Todos os autores apresentam concordância em ser responsável por todos os aspectos do trabalho, no sentido de garantir que as questões relacionadas à exatidão ou à integridade de qualquer parte da obra sejam devidamente investigadas e resolvidas. 